Article

\title{
Development of a Highly Flexible and High-Resolution Deep-Towed Streamer
}

\author{
Toshio Yamazu ${ }^{1}$, Koshi Haraguchi ${ }^{1}\left(\mathbb{D}\right.$, Takahiko Inoue ${ }^{2}$ and Kohsaku Arai ${ }^{2, *(\mathbb{D})}$ \\ 1 Sonic Corporation, 1-18-2 Akebonocho, Tachikawa, Tokyo 190-0012, Japan \\ 2 Geological Survey of Japan (GSJ), The National Institute of Advanced Industrial Science and \\ Technology (AIST), Tsukuba, Ibaraki 305-8567, Japan \\ * Correspondence: ko-arai@aist.go.jp; Tel.: +81-29-861-3822
}

Received: 22 June 2019; Accepted: 31 July 2019; Published: 2 August 2019 updates

\begin{abstract}
We have developed new a deep-towed seismic streamer for sub-bottom profiling. To obtain a high-resolution seismic signal, this streamer can be operated at depths of up to $2000 \mathrm{~m}$, and state-of-the-art technology is adopted with electronics circuits, that can withstand high pressures of up to $22 \mathrm{MPa}$. The streamer houses an ultra-low noise pre-amplifier, micro-processor, AD convertor, high precision clock, gain controller, and other circuitry in an oil-filled vessel. The high $\mathrm{S} / \mathrm{N}$ ratio gives us high-resolution seismic images. The streamer comprises several catenated single modules and the recorded acoustic data are transmitted to the control computer with the Ethernet protocol. This makes the length of the streamer cable, and the number of hydrophones, flexible. For instance, we can use both single-channel and multi-channel streamers in the same system. Up to 24-channels can be catenated.
\end{abstract}

Keywords: deep-towed; seismic streamer; seismic profile; Multi-Channel Seismic (MCS); high-resolution; highly flexible

\section{Introduction}

Multi-channel seismic reflection (MCS) surveys provide one of the most powerful and basic tools for imaging and analyzing the subsurface structure of the sea floor [1]. The MCS system records seismic signals reflected from the surface and subsurface of the bottom of the sea. The vertical and lateral resolutions of the seismic images of the subsurface structure depends largely on the sound source and streamer system. An air gun, boomer, or sparker is normally adopted as an artificial sound source, and the streamer functions as the data acquisition receiver. The vertical resolution is determined by the dominant frequency and bandwidth of the reflected signals [2], and it can be optimized using a high-frequency sound source. De-convolution allows the vertical resolution to be optimized by increasing the bandwidth. The lateral resolution is determined by the size of the Fresnel zone [3], which depends on the dominant frequency of the sound source and the positional relationships among the sound source, the receiver and the reflector, and the sound velocity. If we want to obtain a high-resolution image, the sound source and streamers should be positioned near the sea bottom, as recommended by Bowen [4,5]. Thus high-resolution seismic images of the subsurface, including shallow sediments in a deep-sea environment, cannot be achieved with the conventional near-surface streamer system. To solve the problem by obtaining high-resolution seismic images, a new deep-towed MCS streamer has been developed by Sonic Corporation and the Geological Survey of Japan, National Institute of Advanced Industrial Science and Technology. This study describes the new deep-towed MCS streamer cable in detail. 


\section{System Overview}

The newly developed, deep-towed streamer comprises a data control module, battery module, and multiple, individual, independent acoustic modules, that can be catenated. The number of acoustic modules that can be catenated range from one (single-channel streamer) to 128 (multi-channel streamer), in theory, through data transmission using an Ethernet hub, while, in practice, this is limited to 24 modules. The distance between acoustic modules is flexible and can be set by changing the length of the connection cable. Thus, the operator can determine the number of acoustic modules, and the distance between acoustic modules, to suit the measurement conditions. Figures 1 and 2 show a block diagram of a first trial system with 12 acoustic modules (12-channel streamer) that can monitor a reflected signal in real time using Ethernet protocol.

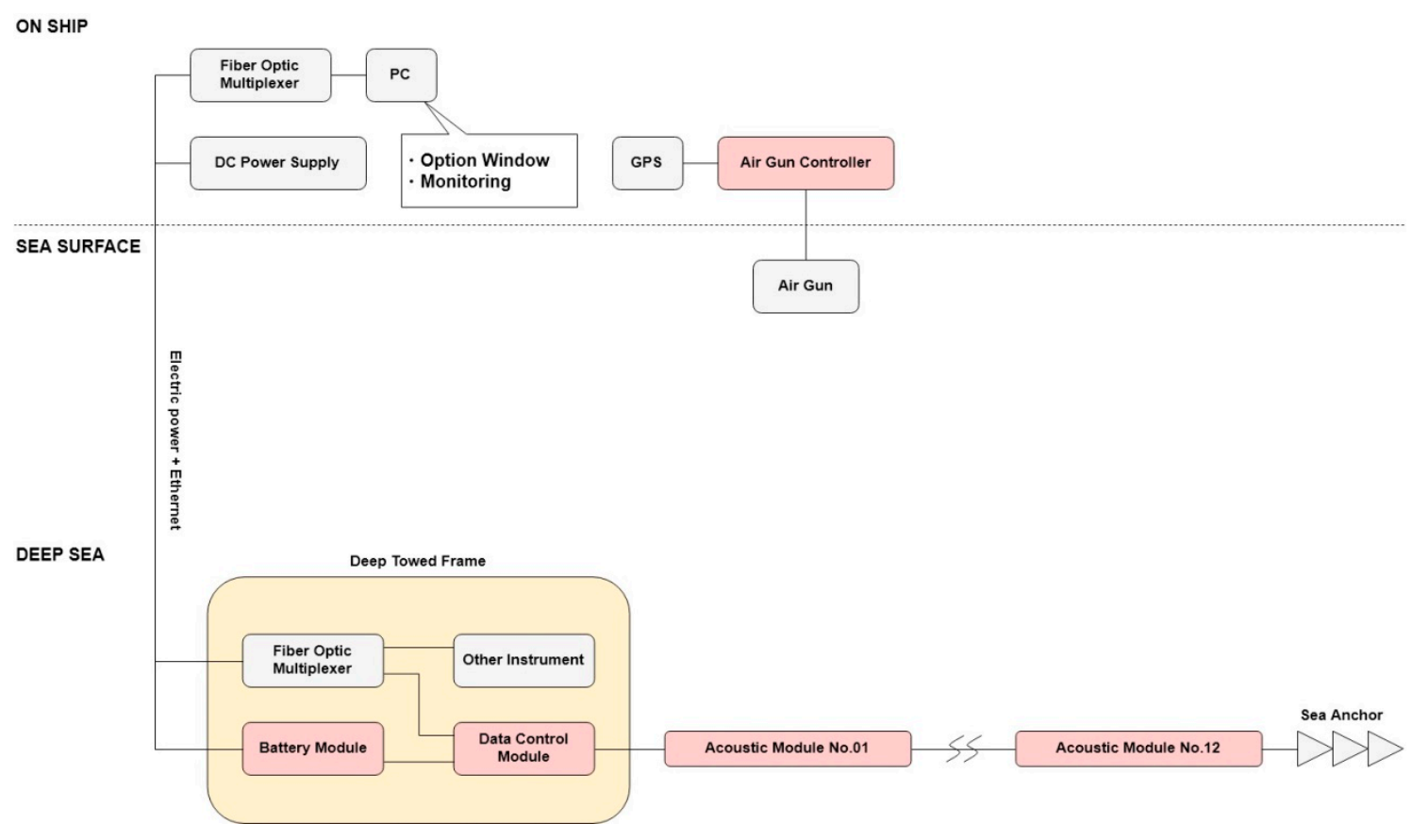

Figure 1. Block diagram of the deep-towed streamer.

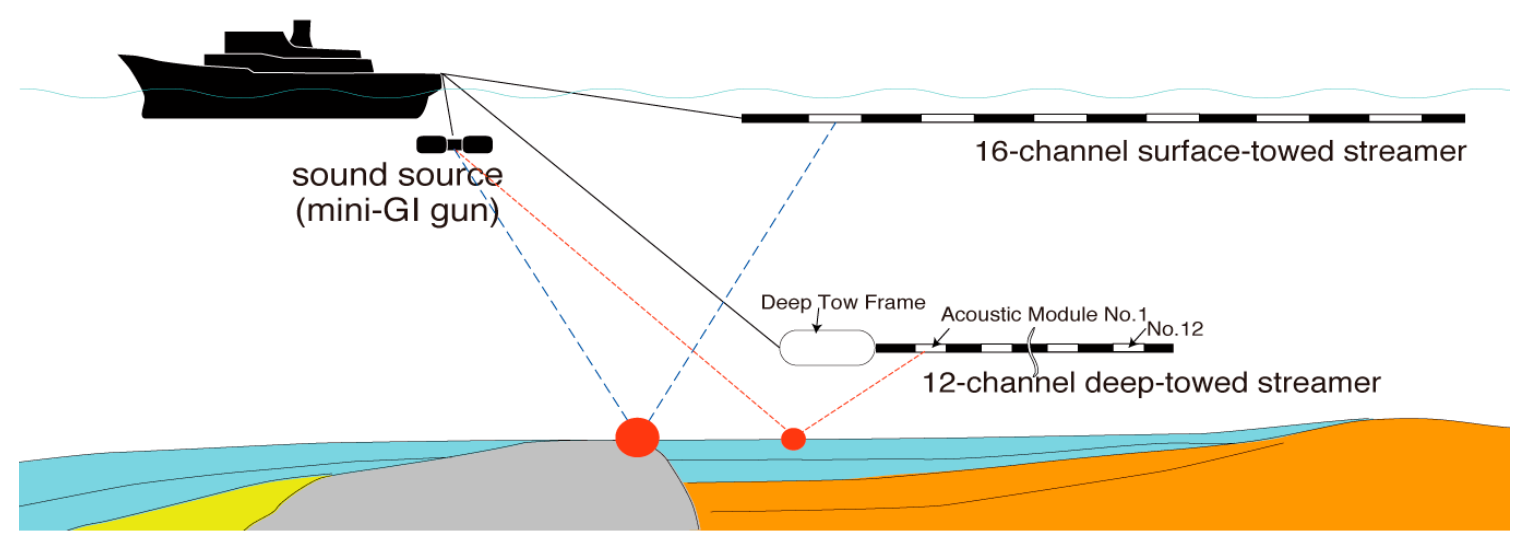

Figure 2. Survey image of a new deep-towed 12-channel trial system, with a surface-towed sound source and existing 16-channel streamer.

This system comprises three parts: The air gun control, monitoring and power supply, and the signal detection part. The air gun control part comprises a chip scale atomic clock (CSAC) with a global positioning system (GPS) signal receiver, an air gun controller that functions as a GPS emulator, and an air gun. The monitoring and power supply part includes, a personal computer (PC) to compile a 
measurement condition table (which includes the gunshot timing and its intervals communicated from the air gun control part), the signal gain, and the coefficient of the band pass filter for noise reduction. The PC monitors all the seismic data that are stored in the data control module as multiplexed data. In this system, the DC power supply is $14.5 \mathrm{~V}$ DC at $3 \mathrm{~A}$. The signal detection part comprises the deep-towed frame and multiple acoustic modules, as shown in Figure 1. In case using an off-line deep-towed frame, seismic signal and depth value are stored in the Data Control Module. In our system, a 2400 DT-2 (EdgeTech, West Wareham, MA, USA) is adopted for the deep-towed frame. The deep-towed frame comprises a battery module, a data control module, a fiber optic multiplexer, and other instruments, such as side scan sonar, including, a position and depth sensor, where the readings are telemetered to the ship in real time. The signal detection part comprises a number of single acoustic modules (multi-channel streamer). The data control module and all the single acoustic modules are connected by an Ethernet/power cable. A sea anchor is fixed to the end of the streamer line to maintain tension on the line.

\section{Description of the Modules}

This section describes the three newly developed main modules. Figure 3 shows a block diagram of the data control module and acoustic module.

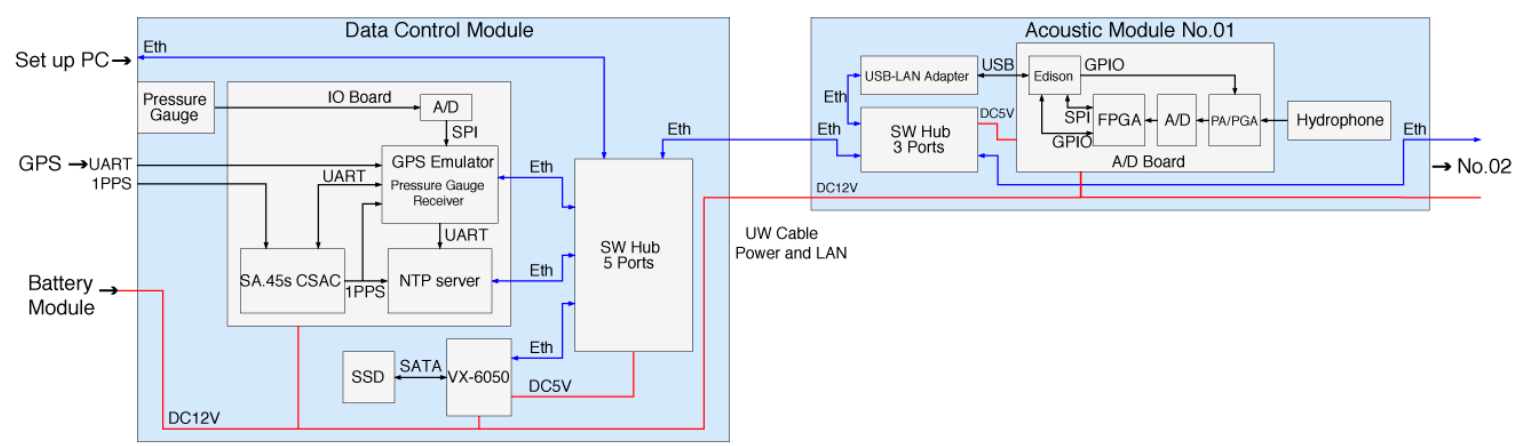

Figure 3. Block diagram of the main modules in this system. Eth: Ethernet, SW Hub: Switching Hub, UW: Under Water.

\subsection{Data Control Module}

The data control module comprises a pressure sensor for detecting the water depth, the CSAC for managing the time interval and the time stamp written with the acquisition data, 2 terabytes of storage (SSD), and computer boards in the pressure vessel for the overall control of the streamer cable and Ethernet hub. The computer motherboard is a VX-6050 with an Intel Atom processor (INNOTECH Corp., Yokohama, Japan).

The CPU motherboard, which runs Linux, controls data acquisition, data storage, measurement time, and measurement conditions. An SA.45sCSAC option 003 (Microsemi Corp., Aliso Viejo, CA, USA) is adopted for the atomic clock. The GPS emulator, formed by the atomic clock and another small CPU, can be linked to GNSS time before system deployment. Time management is executed by this time emulator.

The small CPU also receives pressure data from the pressure gauge, that is installed in the data control module, which is then converted to depth values. For pressure measurements, a high precision Precise line pressure gauge (Keller America, Newport News, VA, USA) with a measurement range of $0-50 \mathrm{MPa} \pm 0.1 \%$ full scale is adopted. The gauge outputs a $0-5 \mathrm{~V}$ signal that corresponds to the pressure range and this is converted to a digital signal by a 16-bit ADC. Time-stamped hydrophone raw data from the acoustic module are stored in the 2-terabyte SSD. Figure 4 shows an external view of the data control module. The titanium high pressure vessel (a diameter of $177 \mathrm{~mm}$ and a length 435 $\mathrm{mm}$ ) is designed to withstand $50 \mathrm{MPa}$ and is pressure-tested up to $22 \mathrm{MPa}$. 


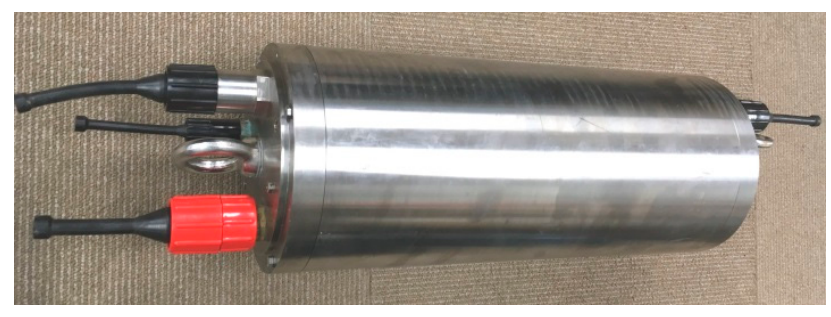

Figure 4. Data control module.

\subsection{Acoustic Module}

The single acoustic module contains dual hydrophones to increase the directionality and sensitivity, a servo-controlled pre-amplifier, with a signal gain of $26 \mathrm{~dB}$, a program-controlled gain amplifier with a signal gain selectable between $0 \mathrm{~dB}$ and $40 \mathrm{~dB}$, an analog-to-digital converter (ADC) with 24-bit resolution, a microcomputer with 1 GB of memory, and a SW Hub/USB-LAN adapter in an oil-filled vessel. The hydrophones are AQ-4 (Teledyne Benthos, North Falmouth, MA, USA). The preamplifier contains a high-pass filter with a stop-band frequency of $5 \mathrm{~Hz}$ and an attenuation of $-20 \mathrm{~dB}$ to reject low-frequency noise generated by fluctuations in the water pressure on the streamer cable. This noise can cause baseline fluctuations that can push the signal over the range of the preamplifier, which will clip it and give a zero output.

The microcomputer an Intel Edison runs Linux, manages data acquisition, the window range and damping rate of the digital noise filter (FIR), the signal amplification level and communication control between the data control module and other units. Each acoustic module has an Ethernet address that can be used to identify the position of each single streamer in the multi-channel streamer cable and its data. As the acoustic module is in an oil-filled vessel, all electronic components are immersed in silicon oil and can withstand pressures up to $22 \mathrm{MPa}$. To obtain a high-pressure resistance for the electronic modules, electronic components, mounted on the circuit boards that were not resistant to high pressures, were treated with a special anti-pressure fabrication. The advantage of this configuration is that it makes the line length of the analog signal from the hydrophone (AD-4) to the preamplifier as short as possible, allowing the analog signal to be converted to a digital signal as soon as possible, thus contributing to an ultra-low noise acoustic detector. The primary gain of the preamplifier is $26 \mathrm{~dB}$ and this is controlled by the gain control amplifier via control software.

Figure 5 shows a schematic diagram of a single acoustic module. The overall length is $985 \mathrm{~mm}$ and the maximum diameter is $142 \mathrm{~mm}$. The length of the main module is $495 \mathrm{~mm}$ and the diameter is $73 \mathrm{~mm}$. The weight is $7.5 \mathrm{~kg}$ in air and $3.7 \mathrm{~kg}$ in sea water. Figure 6 shows a perspective drawing of the single acoustic module. The front and rear sections of this module are enclosed in buoyancy materials.

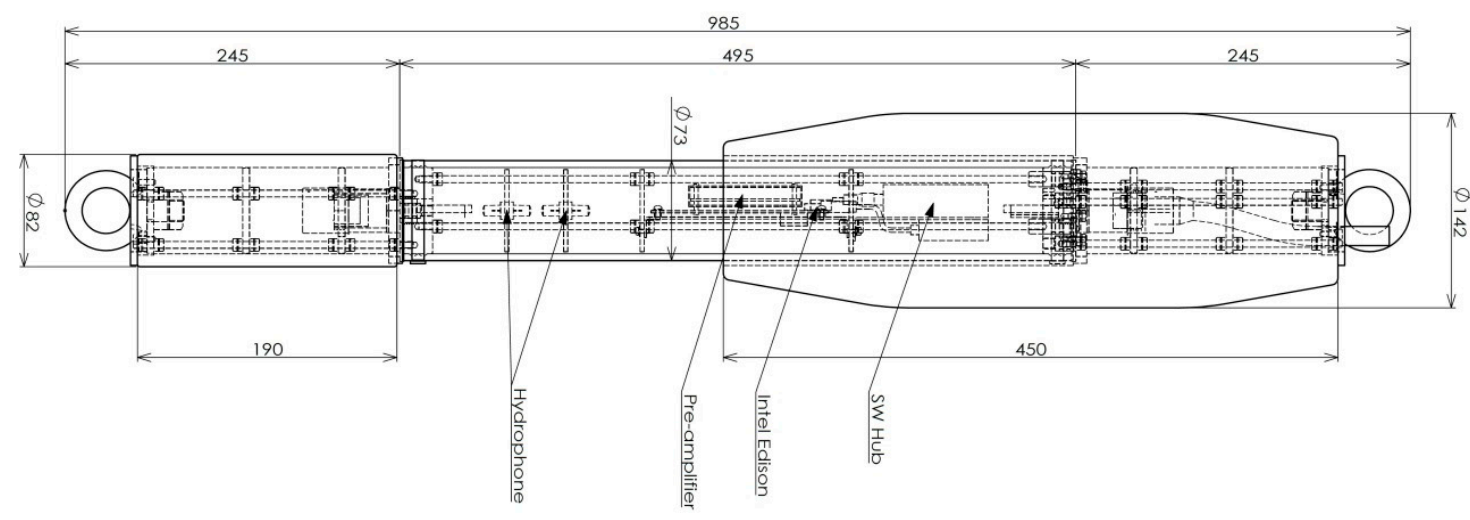

Figure 5. Schematic diagram of the single acoustic module. 


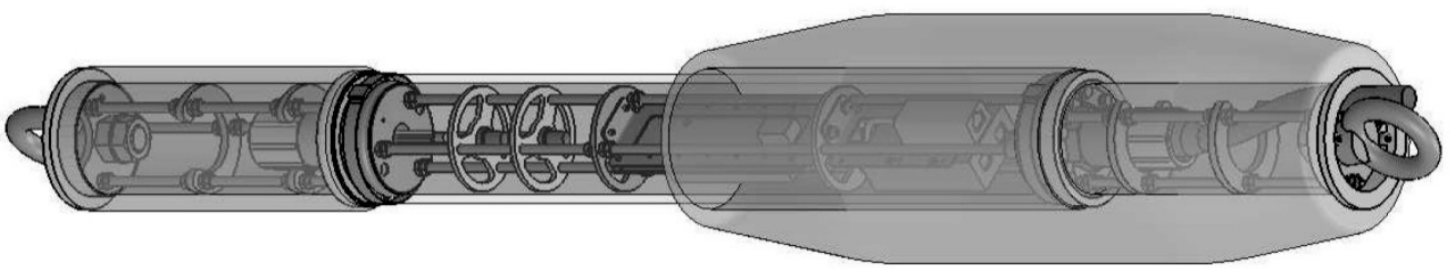

Figure 6. Perspective drawing of the single acoustic module.

Figure 7 shows the acoustic module without its buoyancy materials. The outer tube of the main module is made of urethane resin (OCC Corp, Yokohama, Japan) filled with silicon oil (KF96L-0.65CS, Shin-Etsu Chemical Co., Ltd., Tokyo, Japan) to propagate the signal to the hydrophone. Both ends of the acoustic module have eyebolts for catenating and Ethernet/power connectors. This module can operate in deep water up to a pressure of $22 \mathrm{MPa}$. Figure 8 shows the preliminary setup work on the ship before deployment.

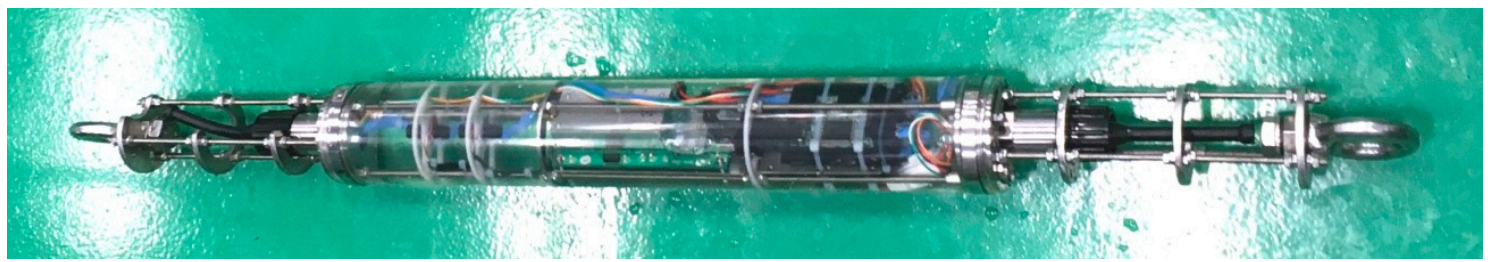

Figure 7. Photograph of an acoustic module without the buoyancy materials.

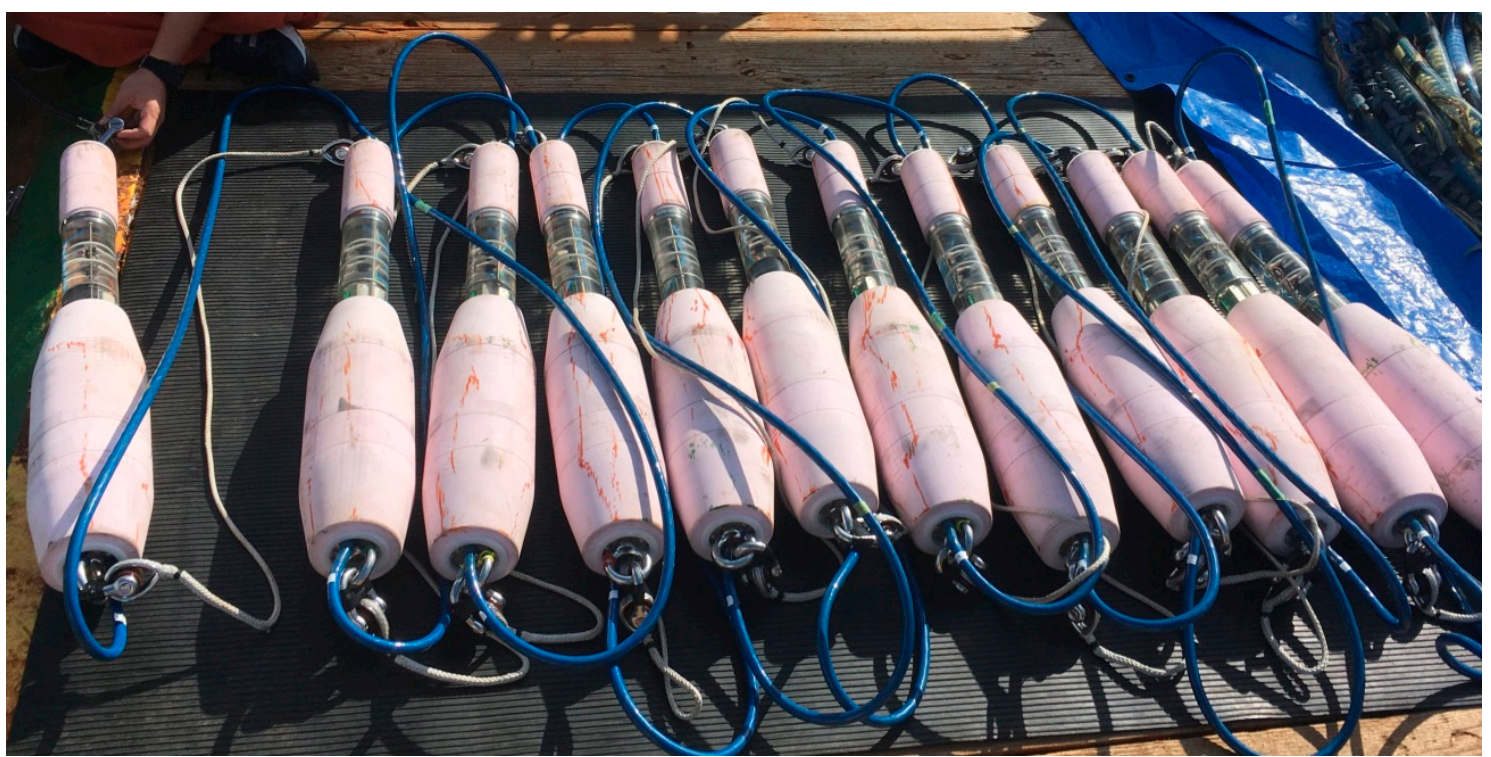

Figure 8. Photograph of 12 catenated acoustic modules with buoyancy materials being prepared for deployment on the ship.

\subsection{Battery Module}

The titanium battery module, which is designed to allow offline operation, contains a 35-Ah battery in a pressure vessel. It has a diameter of $267 \mathrm{~mm}$, a length $355 \mathrm{~mm}$. and weighs $45 \mathrm{~kg}$ in air and $19 \mathrm{~kg}$ in sea water. Battery modules can be catenated to increase capacity and can accept an external electrical input. The battery cell is a lithium ion battery (ALM 12V35, NEC Energy Solutions, Westborough, MA, USA). The pressure vessel is tested up to $22 \mathrm{MPa}$ and designed to withstand up to $50 \mathrm{MPa}$. Two battery modules, installed in a deep-towed frame, will allow $44 \mathrm{~h}$ of continuous operation for a streamer cable with 12 catenated acoustic modules. Figure 9 shows an external view of battery module. 


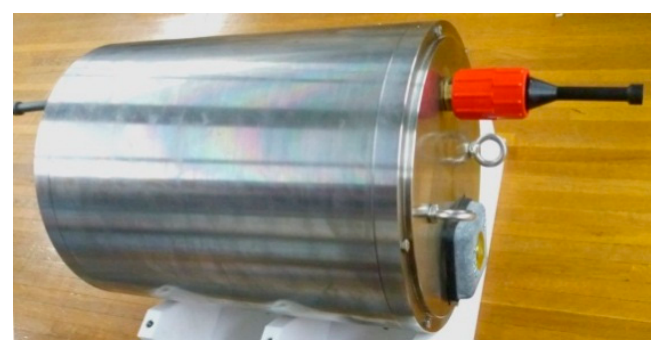

Figure 9. External view of the battery module.

\section{Specifications}

$\diamond$ Frequency response

$\diamond$ Sensitivity

$\diamond$ Depth

$\diamond$ Distance between acoustic modules

$\diamond$ Number of acoustic modules

$\diamond$ Operating time

$\diamond$ Time management

$\diamond$ Amplifier gain

$\diamond$ Resolution power
$5 \mathrm{~Hz}$ to $15 \mathrm{kHz}$

$-201 \mathrm{dBV}$ re $1 \mu \mathrm{Pa}$

$-2200 \mathrm{~m}$

Flexible

Flexible, normally 12, with a min of 1 and a max of 24

$44 \mathrm{~h}$ with two battery modules and 12 acoustic modules with a 14.5 V DC 3 A power supply.

If a $14.5 \mathrm{~V}$ DC 5 A power supply is available, no restrictions on operating time.

Atomic clock with GPS signal receiver

$26 \mathrm{~dB}$ to $66 \mathrm{~dB}$ in 8 steps

$1.2 \mu \mathrm{Pa}$ in theory

\section{Test Data}

The system was tested in the deep sea to a water depth of more than $1000 \mathrm{~m}$, which is located on the slope of the Suruga Trough. Figure 10 shows an example of a reflection image obtained by this system.

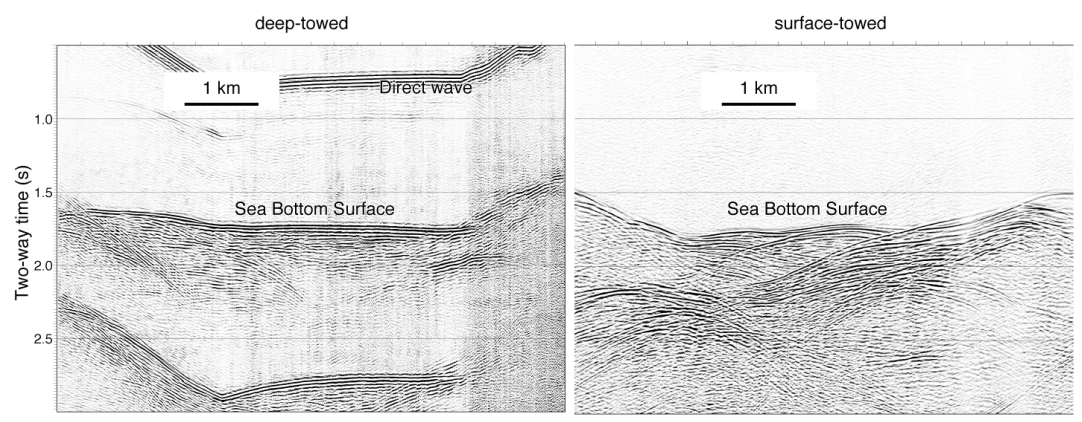

Figure 10. Comparison of the reflection images obtained by new system with surface-towed cable, which is same scaled profiles.

For these data, the depth of the sea bottom is about $1350 \mathrm{~m}$, and towing depth of the streamer is about $600 \mathrm{~m}$. The sound source that was used was a mini-GI gun system (Sercel Inc., Houston, TX, USA), and the volume of both the generator and the injector were $491.6 \mathrm{~cm}^{3}$. Figure 10 shows that the comparison of the reflection images, obtained by this new system, with the existing surface-towed cable. Processing the deep-towed data was carried out using depth correction, 30 to $200 \mathrm{~Hz}$ band-pass filtering, gain control, deconvolution, and brute stacking. The surface-towed 16-channel data was performed using common-midpoint (CMP) sorting, bandpass filtering, gain control, deconvolution, normal move out (NMO), and CMP stacking. Even in the slope area, the surface-towed streamer recorded the arrival of the sound source and assumed that the reflection came from straight below. As a result, the surface strong reflector will appear less steep and shallower shows as noise reflection 
below the sea bottom surface. Our new deep-towed data are less effected by displacements in position. The subsurface stratified sediments are much clear in the reflection images obtained by new system [6].

\section{Summary}

We have developed a new deep-towed streamer, that allows a multi-channel streamer to be configured by catenating a flexible number (up to 24) of single acoustic module physically and electrically, and with a flexible distance between each channel. These deep-towed multi-channel streamers can withstand pressures of up to $22 \mathrm{MPa}$. Single multi-channel streamers can be connected together in an Ethernet network to obtain real-time seismic data. The electrical components in the devices that acquire seismic data in the acoustic module are immersed in silicon oil to withstand high pressures, with the electrical components in the main devices treated with a special anti-pressure fabrication. This shortens the analog signal line length and gives a good $\mathrm{S} / \mathrm{N}$ ratio.

The system was tested in the deep sea and high-resolution seismic data were obtained.

Author Contributions: Conceptualization, T.Y., K.H., T.I., and K.A.; methodology, K.H.; software, T.Y.; investigation, T.I.; data curation, T.I. and K.A.; writing-original draft preparation, T.Y. and K.H.; writing一review and editing, K.A.; project administration, K.A.

Funding: This research received no external funding.

Acknowledgments: This work benefited from the invaluable support of Manabu Matsumoto (Gazougiken Co. Ltd.) in the development of the control software for the system. We thank Michael Luetchford, BTech, from Edanz Group (www.edanzediting.com/as) for editing the English text of a draft of this manuscript.

Conflicts of Interest: The authors declare no conflict of interest.

\section{References}

1. Wood, W.; Gettrust, J.F.; Spychalski, S. A new deep-towed, multi-channel seismic system. Sea Technol. 2003, $44,44-49$.

2. Chapman, N.R.; Gettrust, J.F.; Walia, R.; Hannay, D.; Spence, G.T.; Wood, W.T.; Hyndman, R.D. High Resolution deep-towed multichannel seismic survey of deep sea gas hydrates off western Canada. Geophysics 2002, 67, 1038-1047. [CrossRef]

3. Lindsey, J. The Fresnel zone and its interpretative significance. Lead. Edge 1989, 8, 33-39. [CrossRef]

4. Bowen, A.N. A High-Resolution Seismic Profiling System using a Deep-Towed Horizontal Hydrophone Streamer. Mar. Geophys. Res. 1984, 6, 275-293. [CrossRef]

5. Nouze, H.; Sibuet, J.C.; Savoye, B.; Marsset, B.; Thomas, Y. Pasisar: Performances of a high and very high resolution hybrid deep-towed seismic device. Mar. Geophys. Res. 1997, 19, 379-395. [CrossRef]

6. Inoue, T.; Arai, K.; Yokoyama, S.; Murakami, F.; Nishimura, K.; Yamazu, T.; Haragushi, K. OS13D-1530: High-resolution geological structure in deep sea area obtained by new developing deep towing multi-channel streamer system. In Proceedings of the AGU Fall Meeting, Washington, DC, USA, 10-14 December 2018.

(C) 2019 by the authors. Licensee MDPI, Basel, Switzerland. This article is an open access article distributed under the terms and conditions of the Creative Commons Attribution (CC BY) license (http://creativecommons.org/licenses/by/4.0/). 